\title{
Lost Baryons at Low Redshift
}

\author{
Smita Mathur ${ }^{1}$, Fabrizio Nicastro ${ }^{2}$, and Rik Willimas ${ }^{3}$
}

1 Astronomy Department, The Ohio State University, Columbus, OH 43210

email: smita@astronomy.ohio-state.edu

2 OAR-INAF, Rome, Italy

3 Leiden University, Netherlands

\begin{abstract}
We review our attempts to discover lost baryons at low redshift with the "X-ray forest" of absorption lines from the warm-hot intergalactic medium. We discuss the best evidence to date along the Mrk 421 sightline. We then discuss the missing baryons in the Local Group and the significance of the $\mathrm{z}=0$ absorption systems in X-ray spectra. We argue that the debate over the Galactic vs. extragalactic origin of the $\mathrm{z}=0$ systems is premature as these systems likely contain both components. Observations with next generation X-ray missions such as Constellation-X and XEUS will be crucial to map out the warm-hot intergalactic medium.
\end{abstract}

Keywords. atomic processes, Galaxy: formation, intergalactic medium, quasars: absorption lines, Local Group, cosmology: observations, X-rays: galaxies

\section{Introduction: The Missing Baryons}

Given the title of this symposium, it should not come as a surprise to know that most of the baryons at low redshift are missing; here is how we know. The theory of big-bang nucleosynthesis, together with the observations of the deuterium abundance implies that the ratio of baryon density to the critical density of the Universe is about $4 \%: \Omega_{b} \approx 0.04 h_{70}^{-2}$ (Burles \& Tytler 1998). The concordance cosmology with the new WMAP observations also predicts a similar number for $\Omega_{b}$ (Bennett et al. 2003). This theoretical expectation is consistent with the observations of Lyman $\alpha$ forest at high redshift. At low redshift, however, the Lyman $\alpha$ forest thins out, so the baryons clearly do not stay in the warm, photoionized intergalactic medium at low redshift, as they did at high redshift. Perhaps, as the Universe evolved, and more and more galaxies formed, the baryons were accreted onto the virialized systems. The baryon census at low redshift, however, proved it not to be the case. All the stars and gas in galaxies account for only $10 \%$ of all the baryons (Fukugita, Hogan \& Peebles 1998). Hot gas in clusters of galaxies makes a similar contribution to $\Omega_{b}$. Cold gas at low redshift also adds to the baryon budget, but most of the baryons were found to be lost. This is the so called missing baryon problem.

Cosmological hydrodynamic simulations found that as the Universe evolves, the gas in the intergalactic medium gets shock heated to high temperatures $\left(10^{5}-10^{7} \mathrm{~K}\right)$ and forms a web like large-scale structure of filaments and sheets. Galaxies and clusters of galaxies form in dense knots in the filamentary structure, but even at low redshift most of the baryons still reside in the diffuse intergalactic medium (IGM). The temperature of the IGM is too high to keep hydrogen neutral, which is why Ly $\alpha$ forest thins out. Given the predicted temperature range, the low redshift IGM was called warm-hot intergalactic medium, or WHIM, by Cen \& Ostriker (1999), which is hotter than the warm IGM at high redshift, but cooler than the hot gas in clusters of galaxies. 


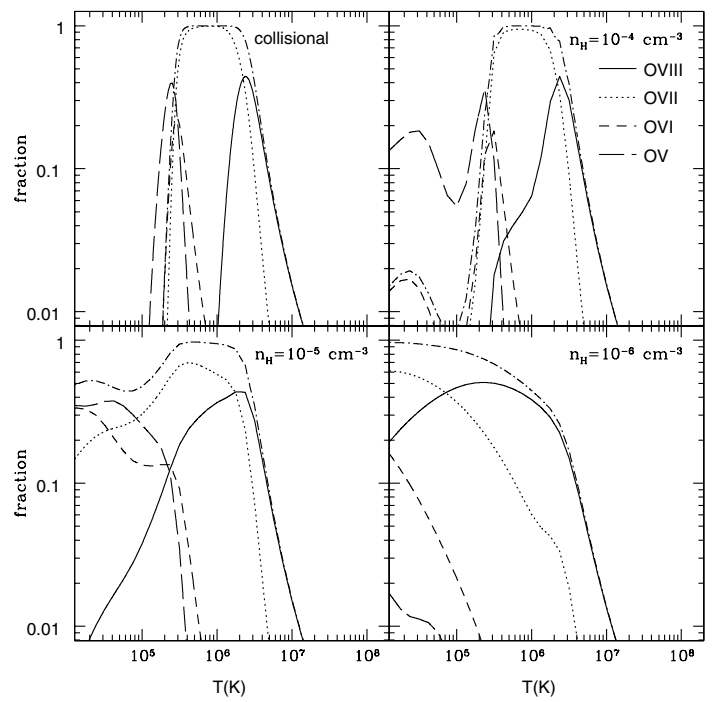

Figure 1. The fraction of oxygen in different ionization states vs. temperature. The top left panel is for the pure collisional ionization while photoionization from metagalactic UV and X-ray background is included in the other panels in which the density is $10^{-4}, 10^{-5}$, and $10^{-6} \mathrm{~cm}^{-3}$ from top right to bottom right.

Even though most of the hydrogen in the WHIM is ionized, most of the metals are not fully ionized. Thus, in spectra of distant quasars, the WHIM may imprint its signature by absorption lines of highly ionized metals. Oxygen being one of the most abundant metals, absorption lines of $\mathrm{O}$ VII are expected to be strongest (see figure 1, adapted from Mathur et al. 2003. O VII is the dominant ionization state of oxygen in the WHIM temperature range).

\section{The Quest for WHIM}

The resonance transitions of the dominant ions fall in the $\mathrm{X}$-ray band-pass. The strongest line of O VII , for example, is at $21.602 \AA$. One thus expects to detect an "X-ray forest" of absorption lines from hydrogen-like and helium-like ions in X-ray spectra of distant quasars. Such an experiment, however, could not be performed before the launch of Chandra and XMM-Newton observatories, which, for the first time, carry gratings on board allowing high resolution spectroscopy. Even then, this is a very difficult experiment because these X-ray telescopes are small and given how faint the quasars are, the S/N of spectra becomes a limiting factor in detecting weak and narrow absorption lines due to WHIM. For this reason we came up with a strategy of observing blazars in their high state, which would provide temporarily bright light beacons.

\subsection{Chandra, FUSE and HST observations of Mrk 421}

One such blazar is Mrk 421 which we monitored with the Rossi X-ray Timing Explorer (RXTE). When the Mrk 421 flux was almost two orders of magnitude larger than its quiescent flux, we triggered a target-of-opportunity (TOO) observation with Chandra low energy transmission grating (LETG). Thus we obtained the highest S/N grating spectrum among all targets ever observed with Chandra (see figure 2, adapted from Nicastro et al. $2005 \mathrm{a}, \mathrm{b})$. The black dots with error bars are the data and the blue line running through 

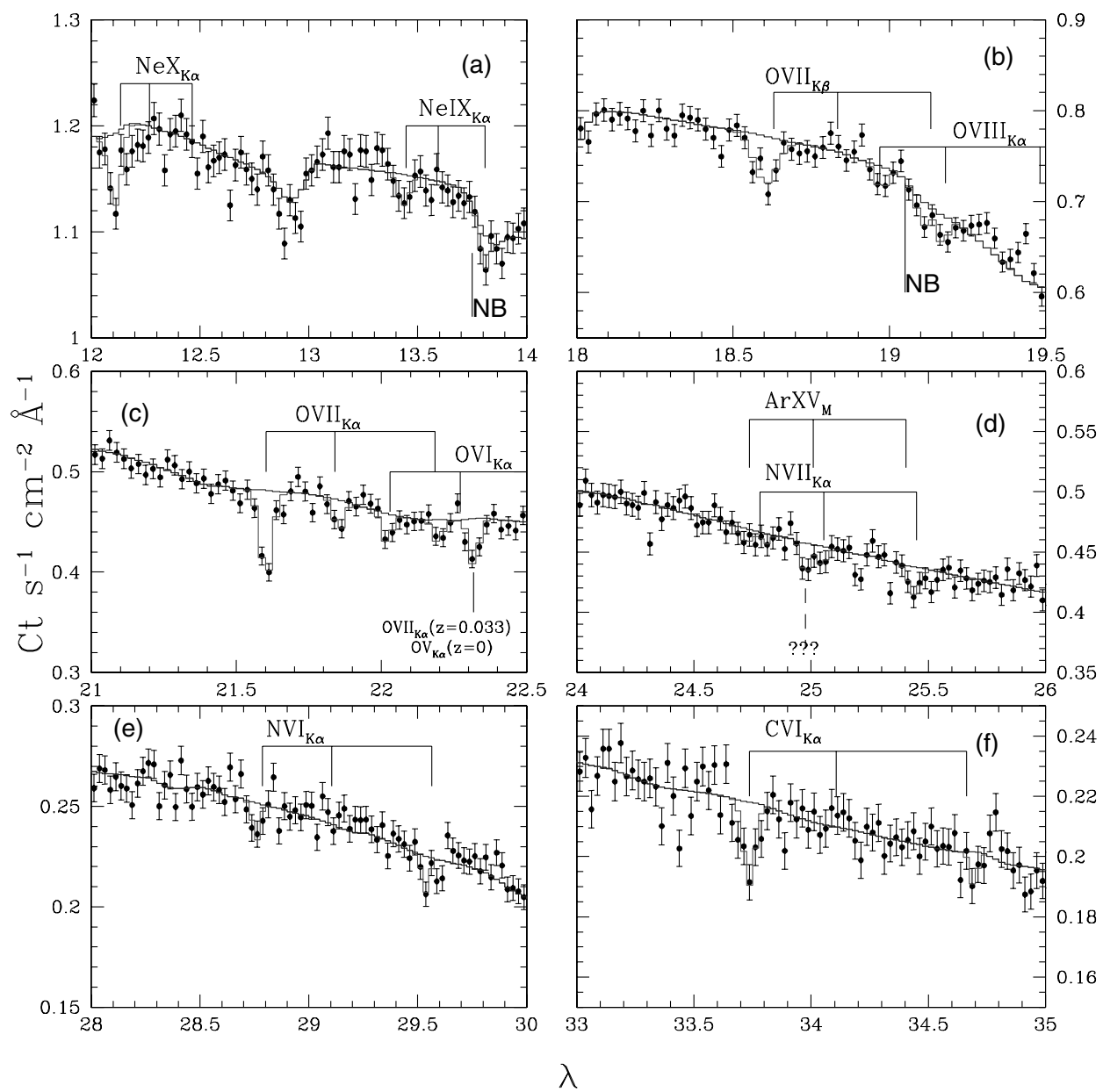

Figure 2. The Chandra LETG spectrum of Mrk 421. Note the detection of absorption lines from various $\mathrm{H}$-like and He-line ions at redshift zero and at $\mathrm{z}=0.011$ and $\mathrm{z}=0.027$ marked with vertical bars.

the spectrum is the Mrk 421 continuum folded with the instrumental response. The red lines mark the absorption lines in the spectrum which we identify with transitions of several ions at redshifts zero, 0.011 and 0.027 . The ions are labeled and the three redshifts are marked with vertical bars. The middle left panel shows the strong transition of $\mathrm{O}$ vII $\mathrm{K} \alpha$ which is clearly detected at all the three redshifts. The strongest line is at $\mathrm{z}=0$, and we will come to this in a later section.

We also observed Mrk 421 with FUSE to look for any possible absorption lines of O VI. The FUSE spectrum, shown in figure 3 (adapted from Nicastro et al. 2005b), is rich with absorption lines from the interstellar medium of the Galaxy. However, O vi absorption lines from the two intervening X-ray absorption systems are not detected.

Mrk 421 was also observed with HST and a portion of the STIS spectrum is shown in figure 4 (adapted from Nicastro et al. 2005b). Again, we do not detect HI Ly $\alpha$ absorption from the two intervening X-ray systems.

To summarize, we detect two intervening absorption systems toward Mrk 421. The $\mathrm{z}=0.011$ system is detected through strong absorption lines of O VII and N VII, though 


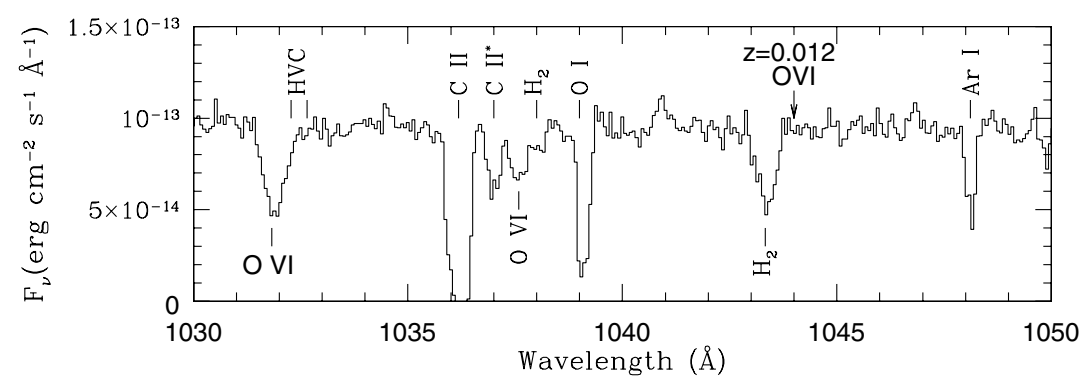

Figure 3. The FUSE spectrum of Mrk 421. No O vi absorption lines are detected at the redshifts of intervening X-ray absorption systems.

$\mathrm{O}$ VI and $\mathrm{H}$ I are not detected. The $\mathrm{z}=0.027$ system is detected through strong absorption lines of O viI, C vi, Nvi, NvII and less significant lines of Ne IX and O VII K $\beta$. O VI is not detected in this systems as well and $\mathrm{H}$ I detection is also not strong, if at all present.

With the column density of detected ions and the upper limits obtained from nondetections we determine the physical properties of the absorbing gas. The method can be understood from figure 1 in which fractional ionization of oxygen is plotted as a function of temperature. The top left panel corresponds to pure collisional ionization; we see that the fraction of oxygen in a given ionization state is a strong function of temperature and higher ionization states reach their peak fraction at higher temperatures. Thus, the column density ratios of two ions depend upon temperature.

In the other three panels of figure 1, photoionization from metagalactic UV and X-ray backgrounds is also included in calculating ionization fractions. As expected, the shapes of the curves change when photoionization is added. The three panels correspond to three different densities of the IGM, which are affected by photoionization to different degrees. Thus, the ionic ratios become not only dependent on the temperature, but also on the density of the IGM. In particular, we find that the O vII/O vi ratio is a sensitive probe of the temperature of the IGM, while the O VIII/O vi ratio measures the density (Mathur et al. 2003). This can be understood intuitively; if a system contains both O vIII and O VI with measurable amounts, then photoionization must play a role, and lower the density, higher the effect of the photoionization.

We can then make figures similar to figure 1 for other elements and generate expected values of column density ratios for a variety of ions. We can then compare our observed line ratios with the theoretical calculations to constrain the properties of the intervening systems. One such diagnostic plot is shown in figure 5 (adapted from Nicastro et al. $2005 \mathrm{~b}$ ) in which various ion ratios are plotted vs. temperature. The thin curves represent the theoretical models while the thick curves represent observed values within $1 \sigma$ errors. All the observations overlap in the temperature range of $0.4-3.3 \times 10^{6} \mathrm{~K}$.

Strong detection of oxygen and non-detection of $\mathrm{HI}$ allow us to put a constraint on the abundance ratio to be $[\mathrm{O} / \mathrm{H}] \sim-1.46$, a reasonable value for the WHIM. The total equivalent column density is $\mathrm{N}_{H}=0.3-7.3 \times 10^{19} \times 10^{[O / H]_{-1}} \mathrm{~cm}^{-2}$. We have no direct constraint on the density, but if it of the order of $10^{-5} \mathrm{~cm}^{-3}$, then the inferred path length 


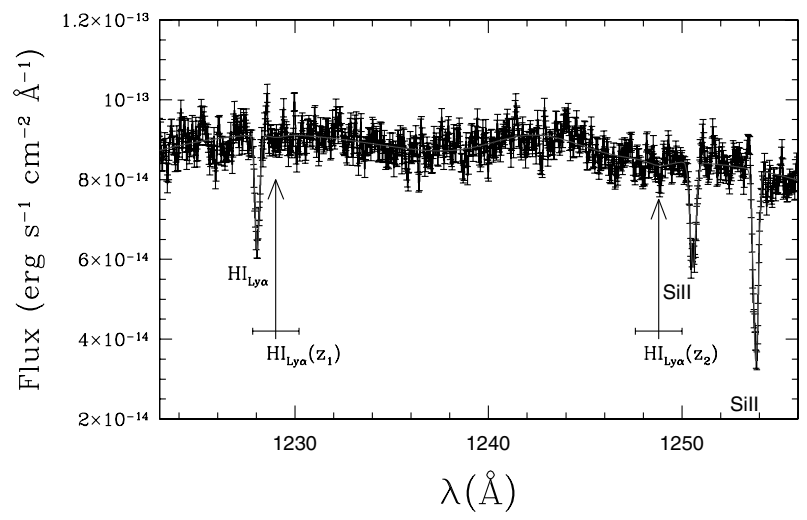

Figure 4. The HST spectrum of Mrk 421 showing no HI absorption from the two intervening X-ray systems

is of the order of a Mpc: $\mathrm{D}=0.1-2.4 \times 10^{[O / H]_{-1}}\left(n_{-5}\right)^{-1}$. Thus all the inferred parameters of the absorption systems are consistent with those of the WHIM.

We have detected two intervening O viI systems in the pathlength out to Mrk 421. This is just one sightline and there are only two systems in it, so we are in the regime of low number statistics, but nonetheless we can calculate the number density of $\mathrm{O}$ VII filaments, albeit with a large error bar. In figure 6 we compare this with the theoretical curve and again find consistency. The theoretical curve in new simulations (Cen \& Fang 2006) has lower normalization, but the Mrk 421 data point is still consistent with models within $2 \sigma$.

\subsection{Lost is Found?}

Given the consistency of all the observed parameters with the theoretical expectations for the WHIM, it is reasonable to assume that the observed systems are indeed WHIM filaments. We can then calculate the baryon density associated with these systems and compare it with the missing baryons. We find

$$
\Omega_{b}=0.032_{-0.021}^{+0.042} \times 10^{-[O / H]_{-1}}
$$

consistent with the missing $\Omega_{b}$. We are, however, cautious about claiming that the lost baryons have been found because, once again, the errors on our number are large due to small number statistics. It is therefore imperative to observe more sightlines and detect more WHIM systems. We observed a sightline toward 1ES1028 + 511 with XMMNewton and have tentative detections of a couple of more systems. 1ES1028 +511 is at a higher redshift $(\mathrm{z}=0.361)$ than Mrk $421(\mathrm{z}=0.03)$, resulting in a smaller number density of O VII systems along this sightline. Note, however, that the column density threshold in the XMM-Newton spectrum of 1ES1028 + 511 is much larger and the observed number density is still consistent with the theoretical curve in figure 6. If these are confirmed, then our solitary point on the curve in figure 6 will have company and the errors on $\Omega_{b}$ will be lowered.

\section{The $z=0$ Absorption Systems}

In high quality spectra of several extragalactic sources, absorption lines of O viI K $\alpha$ are detected at redshift zero (see figure 2 for the $\mathrm{z}=0 \mathrm{O}$ VII line in the Mrk 421 sightline). 


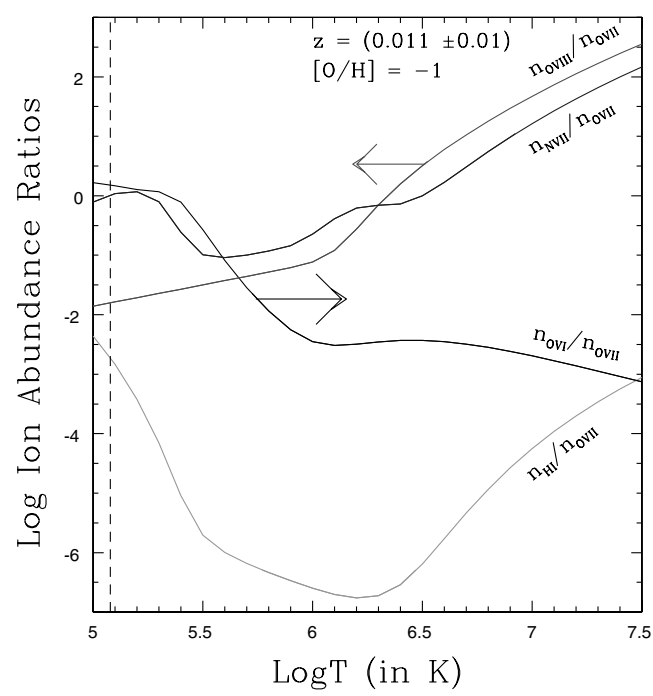

Figure 5. Constraints on the $z=0.011$ system from observed line ratios. The thin lines are the theoretical models while the thick portions on the lines mark observed values with errors. The temperature range where all observations overlap is around $10^{6} \mathrm{~K}$.

The origin of such absorption systems is debated in the literature; is it from the ISM or the halo of our own galaxy or is it from larger scale structures such as the Local Group? It should be noted that O VI absorption is also ubiquitous in extragalactic sightlines observed by FUSE. One normally detects a broad component of O vi at redshift zero from the thick disk of our own Galaxy and often an additional component at a velocity offset of more than $100 \mathrm{~km} / \mathrm{s}$ from the so called high velocity cloud. The spectral resolution in the X-ray band is not high enough to resolve the components seen in the FUV. It is not immediately obvious, therefore, whether the OVII line is related to any of the O VI components. The answer to this question is related to the origin of O VII systems.

\subsection{Galactic or Local Group?}

This has become such an important question because we would like to know the associated mass. If the $\mathrm{z}=0$ systems are from the local large scale structure, then the associated mass can be huge. It may even account for the missing baryonic mass in the Local Group. On the other hand, if the $\mathrm{z}=0 \mathrm{O}$ VII systems are from the halo of our Galaxy, then they may carry insignificant amount of mass.

Theoretically, some models of galaxy formation and evolution predict hot halos around galaxies. Similarly, as mentioned in $\S 1$, in the cosmological simulations galaxies form in dense knots in IGM filaments, so we expect to find a local WHIM filament(s) in which our Galaxy is embedded. This expectation was further confirmed with constrained simulations of the local volume (Kravtsov et al. 2002) which show large reservoirs of O VII gas in extended structures around the Galaxy. Thus, understanding the origin of O vil systems will help test the models of galaxy formation and/or large scale structure.

We have been investigating this question systematically (Nicastro et al. 2002, Williams et al. 2005, 2006b, 2007) by modeling the observed O VII and O vi systems. We find interesting differences among different sightlines implying complexity in the structure surrounding the Galaxy. We could not determine the origin of the O viI systems; neither Galactic nor extragalactic origins could be ruled out. One conclusion that we could reach 


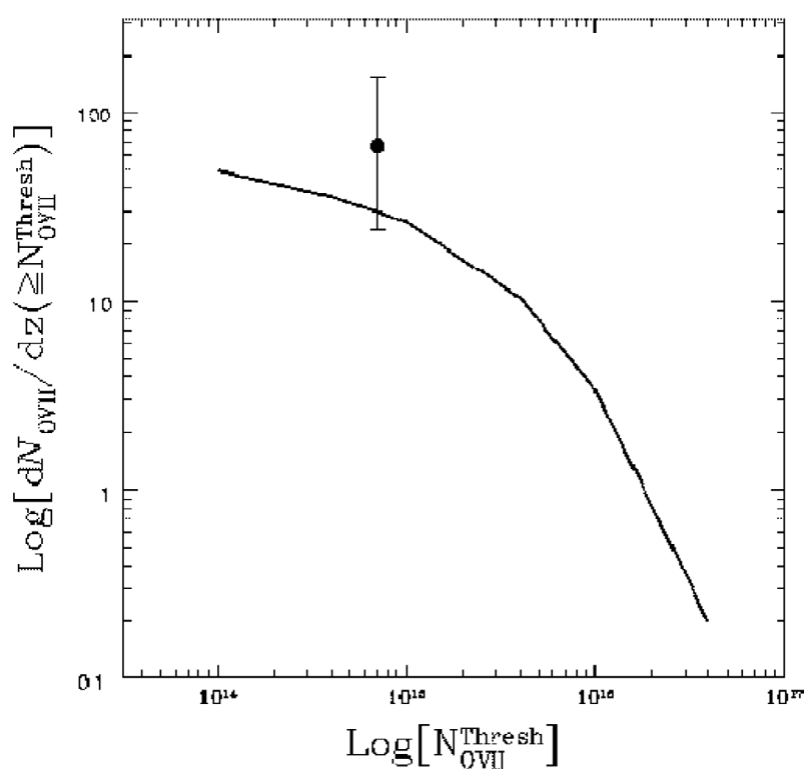

Figure 6. The cumulative number density of O VII filaments due to WHIM is plotted as a function of observational column density threshold. The theoretical curve is from Fang et al. 2002 and the data point with error bars is from our observations.

is that the $\mathrm{O}$ VII absorption lines do not arise in the gas producing the O vI systems. This is most directly seen in the Mrk 279 sightline (figure 7, adapted from Williams et al. 2006) where we found a velocity offset between the O VII line and the O VI HVC.

To summarize, O VII absorption is not associated with any single O VI component. Neither Galactic or extragalactic origin of the O viI systems can be ruled out. Most likely, a variety of phenomena are responsible for the O VII systems as indicated by the differences in their "b" values (velocity dispersion parameters). Better, higher resolution, data are crucial in understanding the origin of O VII systems.

\section{Conclusions \& Future Prospects}

As mentioned by C. Frenk (these proceedings) the claims of the discovery of WHIM have been controversial. The best observed evidence, nonetheless, is from the Mrk 421 sightline in which the chance probability of detecting the observed absorption features by statistical fluke is less than $1 \%$. Detections with higher significance are desirable, of course. Given the importance of the WHIM discovery, we need to observe more sigh-lines and solidly detect at least a few more X-ray forest systems before counting the baryons. The community needs to support observing programs with these goals. It is worth noting at this point that because of the viewing angle constraints on Chandra observations, TOO observations of blazars in high state is not a viable strategy any more.

Even if we succeed in our effort to detect WHIM with Chandra or XMM-Newton, we will be only observing the tip of the iceberg (see figure 8). To map out the WHIM filaments at low redshift, by going down to the column density thresholds much more representative of the large scale structure, Constellation-X and XEUS will be essential. These are future missions of NASA and ESA respectively; mapping the low redshift WHIM is one of their major science drivers. We need to obtain a definite proof of concept with Chandra and $X M M-N e w t o n$ before these mission concepts are finalized. 


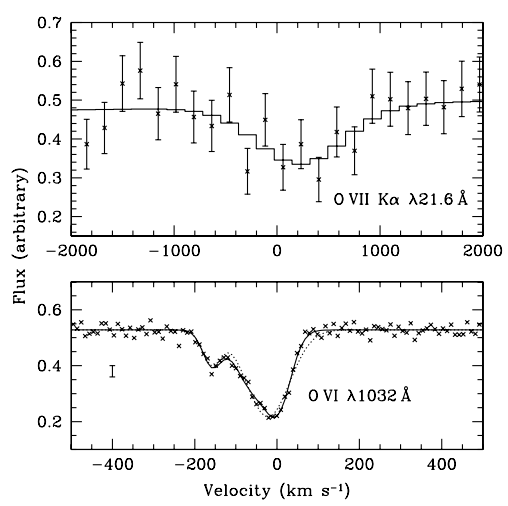

Figure 7. The X-ray and UV z $=0$ systems toward Mrk 279 show velocity offset

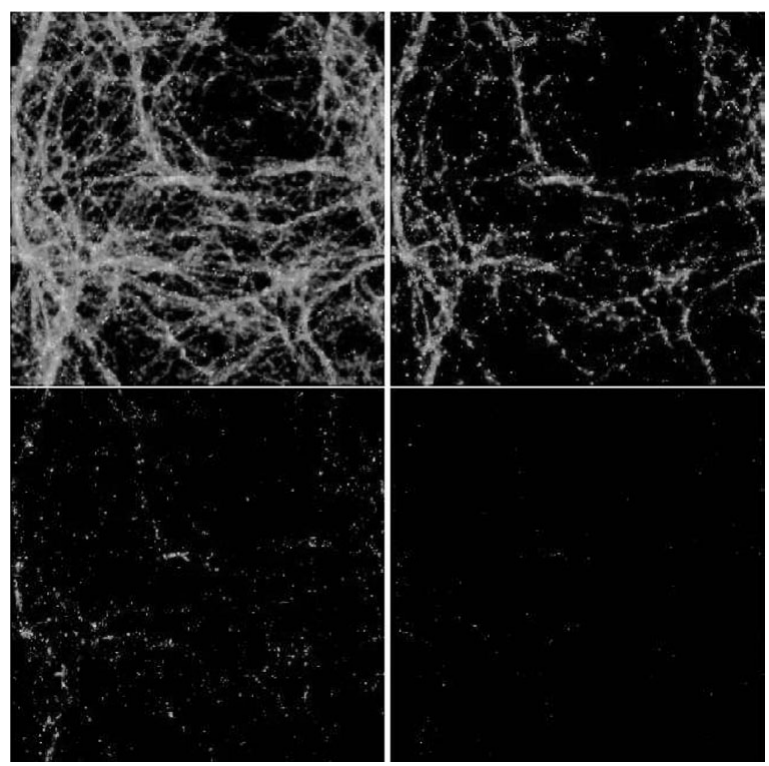

Figure 8. The O VII column density map from the cosmological hydrodynamic simulation of the WHIM (from Chen et al. 2003). The top left panel has a column density threshold of $10^{14} \mathrm{~cm}^{-2}$. The other panels from top right to bottom right correspond roughly to detection thresholds of XEUS, Constellation-X, and Chandra /XMM-Newton respectively (assuming metallicity of a tenth solar).

The science of the $\mathrm{z}=0 \mathrm{O}$ VII systems is equally interesting which bears directly on the models of galaxy formation and/or models of large scale structures. The spectral resolution offered by Chandra and XMM-Newton gratings is just not good enough to understand the origin of these systems. The debate over the Galactic vs. extragalactic origin of O vII systems is premature because we do not have data quality to resolve the issue. Given what we understand from other poor groups similar to the Local Group, the O viI systems must contain both of these components. We propose that the O viI lines contain two (or more) components (Mathur et al. 2007) which will be resolved in higher resolution spectra. Future higher resolution observations are crucial to test this prediction and to make significant progress in this field. 


\section{Acknowledgements}

This work is supported in part by the Chandra grant AR5-6017X.

\section{References}

Bennett, C. L. et al. 2003, ApJS 148, 1

Burles, S. \& Tytler, D. 1998, Space Science Reviews 84, 65

Cen, R. \& Ostriker, J. 1999, ApJ 514, 1

Cen, R. \& Fang, T. 2006, ApJ 650, 573

Chen, X., Weinberg, D., Katz, N. \& Dave, R. 2003, ApJ 594, 42

Fang, T., Bryan, G.L. \& Canizares, C. 2002, ApJ 564, 604

Fukugita, M., Hogan, C. \& Peebles, P. J. E. 1998, ApJ 503, 518

Kravtsov, A., Klypin, A. \& Hoffman, Y. 2002 ApJ 571, 563

Mathur, S., Weinberg, D. \& Chen, X. 2003, ApJ 582, 82

Mathur, S., Williams, R. \& Nicastro, F. 2007, in preparation

Nicastro, F. et al. 2002 ApJ 573, 157

Nicastro, F. et al. 2005a Nature 433, 495

Nicastro, F. et al. 2005b ApJ 629, 700

Williams, R. J., et al. 2005 ApJ 631, 856

Williams, R. J., Mathur, S. \& Nicastro, F. 2006a ApJL 642, 95

Williams, R. J., Mathur, S. \& Nicastro, F. 2006b ApJ 645, 179

Williams, R. J., et al. $2007 \mathrm{ApJ}$ in press.

\section{Discussion}

Bomans: The intervening absorption lines in the Chandra spectrum of Mrk 421 are not detected with XMM-Newton. Could you comment on that?

MATHuR: Because of the higher $\mathrm{S} / \mathrm{N}$ in $X M M$-Newton spectrum, it was natural to expect that the Chandra lines will be detected in the $X M M-N e w t o n$ spectrum. However, S/N is not the only factor in deciding the line detection threshold. The shape of the line response function makes a big difference, just the same way a point spread function affects the point source detection in imaging observations. The line spread function of $X M M$-Newton gratings has wings, increasing the equivalent width limit of line detections. There are a couple of more factors, which we have detailed in Williams et al. 2006a. The bottom line is that XMM-Newton non-detections are consistent with Chandra detections. Later, Rasmussen et al. re-analyzed the XMM-Newton data. Their upper limits are again consistent with Chandra detections, given the values in the paper, even though the text of their paper claims inconsistency!

SCHAYE: We do not know the metallicity of the WHIM, so we can never know whether the missing baryons are found. It would be much more fruitful to assume that the missing baryons are found and use that to constrain the UV and X-ray background

MATHuR: Good point! However, the errors on the observations at present are too large to constrain the background parameters. Secondly, if we actually detect hydrogen with UV spectroscopy from the WHIM systems, then we can measure metallicity. 